See discussions, stats, and author profiles for this publication at: https://www.researchgate.net/publication/328990505

\title{
Isotropic-Resolution Tomographic Diffractive Microscopy
}

Conference Paper · July 2018

DOI: 10.1109/ICTON.2018.8473709

\section{CITATION}

1

9 authors, including:

Matthieu Debailleul

Université de Haute-Alsace

32 PUBLICATIONS 482 CITATIONS

SEE PROFILE

(5)

Jonathan Bailleul

17 PUBLICATIONS 92 CITATIONS

SEE PROFILE

Some of the authors of this publication are also working on these related projects:

Software engineering View project

Project Tomographic Diffractive Microscopy View project
Carole Ecoffet

French National Centre for Scientific Research

58 PUBLICATIONS 677 CITATIONS

SEE PROFILE

Hui Liu

Institute for Advanced Technology in Life Sciences

8 PUBLICATIONS 68 CITATIONS

SEE PROFILE 


\title{
Isotropic-Resolution Tomographic Diffractive Microscopy
}

\author{
Bertrand Simon $^{1 *}$ Member IEEE, Matthieu Debailleul $^{2}$, Mounir Houkal ${ }^{3}$, Carole Ecoffet ${ }^{3}$, Jonathan \\ Bailleul $^{2}$, Joël Lambert ${ }^{2}$, Arnaud Spangenberg ${ }^{3}$, Hui Liu ${ }^{4}$, Olivier Soppera ${ }^{3}$ and Olivier Haeberlé ${ }^{2}$ \\ ${ }^{1}$ Laboratoire Photonique, Numérique et nanosciences LP2N, Institut d'Optique Graduate School, Bordeaux \\ ${ }^{2}$ Institut de Recherche en Informatique, Automatique, Automatique et Signal IRIMAS - EA7499 \\ Université de Haute-Alsace (UHA), 61 rue Albert Camus F-68093 Mulhouse Cedex, France \\ ${ }^{3}$ Institut de Science des Matériaux de Mulhouse IS2M, Université de Haute-Alsace (UHA), CNRS UMR 7361,15 \\ rue Jean Starcky, BP 2488, F-68057 Mulhouse Cedex, France \\ ${ }^{4}$ Institut de Génétique et de Biologie Moléculaire et Cellulaire IGBMC, Université de Strasbourg (UniStra), \\ CNRS UMR 7104, INSERM U964, 1 rue Laurent Fries, F-67404 Illkirch Cedex, France \\ e-mail: bertrand.simon@institutoptique.fr
}

\begin{abstract}
Microscopy techniques based on recording of the optical field diffracted by the specimen, in amplitude and phase, like Digital Holographic Microscopy (DHM) have been a growing research topic in recent years. Tomographic acquisitions are possible if one is able to record information, while controlling variations of the specimen illumination. Classical approaches consider either illumination variation, simple to implement, but suffering fro the classical "missing cone" problem, or sample rotation, delivering images with quasi-isotropic, but lower resolution. We have developed an original-, combined tomographic diffractive microscope setup, making use of specimen rotation as well as illumination rotation, which is able to deliver images with an almost isotropic resolution better than $200 \mathrm{~nm}$.
\end{abstract}

Keywords: 3-D microscopy; Interference microscopy; Digital holography; Holographic interferometry.

\section{INTRODUCTION}

When one does not want to, or cannot use fluorescence microscopy, imaging capabilities of conventional optical microscopes are limited. Various techniques have been developed to image non-fluorescent samples, among them, phase microscopy, which has already found applications in biology and in material science [1]-[5].

Tomographic diffractive microscopy (TDM) (see Ref. [6] for a general introduction to this technique) combines amplitude and phase imaging, together with controlled variations of the conditions of illumination of the sample. A 3-D image of the observed specimen is then obtained by computer-assisted image reconstruction. For weakly scattering specimens, considering that the first Born approximation is valid, the diffracted field is then directly linked to the 3-D Fourier transform of the specimen index of refraction distribution [7].

\section{PRINCIPLE OF ISOTROPIC RESOLUTION TDM}

Figure 1 details the principles of transmission holographic microscopy (DHMs) and tomographic configurations (TDMs). In Fourier domain representing the spatial frequencies of objects of interests, the diffracted field is represented by the $\mathrm{k}_{\text {diff }}$ wavevectors. The $\mathrm{k}_{\text {diff }}$ wavevectors depict the so-called Ewald sphere. Holographic microscopy makes use of only one direction of illumination (usually parallel to the optical axis, and depicted by $\mathrm{k}_{\mathrm{ill}}$ vector). Because of the limited numerical aperture (NA) of the detection objective, only a cap of sphere of $\mathrm{k}_{\text {diff }}$ vectors can indeed be captured (Fig. 1(a)). Thanks to the elastic scattering condition, the registered field is simply linked to specimen frequencies by momentum conservation $\mathrm{k}_{\mathrm{obj}}=\mathrm{k}_{\mathrm{diff}}-\mathrm{k}_{\mathrm{ill}}$. Note that the lateral extension of the $\mathrm{k}_{\mathrm{obj}}$ cap of sphere will provide a good lateral resolution, but its axial extension is very restricted, which translates into poor the imaging capabilities along the optical axis (weak optical sectioning) [8]-[12].

To capture higher object frequencies, one uses inclined illumination, as depicted on Fig. 1(b). When many illuminations are sequentially used (Fig. 1(c)), one obtains an extended and filled support for the Optical Transfer Function (OTF), which translates into better lateral and axial resolutions. However, as for any transmission microscope, the OTF along the optical axis is of lesser extension, and a cone of missing frequencies remains. This translates into lower axial resolution, and also limits sectioning capabilities [10][11].

The second classical technique for TDM makes use of a sample rotation. In that configuration, an almost complete spherical OTF is obtained (Fig. 1(d)). It is however of smaller extension, and anyway, a small set of missing frequencies also still remains along the rotation axis [13][14]. Furthermore, efficient imaging in this configuration requires a large number of sample rotations [15][16].

It has been theoretically proposed [14], to simultaneously obtain improved- and isotropic-resolution images, to combine both techniques of illumination- and specimen rotation. Figure 1(e) shows the final extended OTF support for 1,2 and 4 views. 


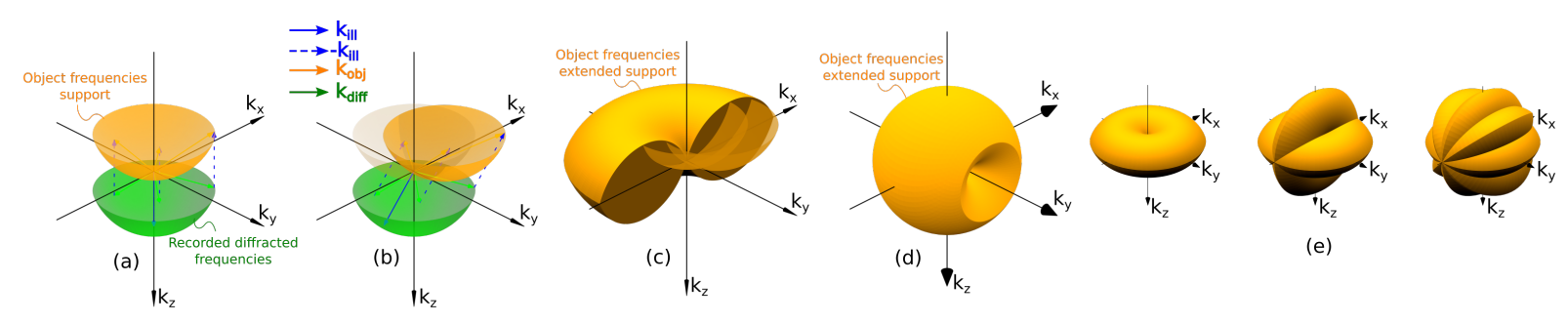

Figure 1. Principle of isotropic resolution TDM (see Ref. [17])

Comparing the final frequency supports is easy, and gives hint about the imaging capabilities of the four DHM and TDMs variants, whose OTFs are presented on Figure 1. In particular, lateral and axial extensions of the frequency support for DHM are [8]:

$$
\Delta v_{x, y}^{\text {Holo }}=\frac{2 n \sin q}{\lambda} \Delta v_{z}^{\text {Holo }}=\frac{n(1-\cos q)}{\lambda}
$$

For TDM with illumination rotation (TDM-IR), the lateral frequency support extension is doubled [8]:

$$
\Delta v_{x, y}^{\mathrm{TDM}-\mathrm{IR}}=\frac{4 n \sin \theta}{\lambda} \Delta v_{z}^{\mathrm{TDM}-\mathrm{IR}}=\frac{2 n(1-\cos \theta)}{\lambda}
$$

For TDM with sample rotation (TDM-SR), almost a complete sphere is obtained for high-NA detection [14]:

$$
\Delta v_{x, z}^{\mathrm{TDM}-\mathrm{SR}}=\frac{4 n \sin (\theta / 2)}{\lambda} \Delta v_{y}^{\mathrm{TDM}-\mathrm{SR}}=\frac{2 n \sin \theta}{\lambda}
$$

And when combining both approaches (TDM-IRSR), one obtains a completely filled support, of same extension than for (TDM-IR), but now fully isotropic, so a sphere:

$$
\Delta v_{x, y, z}^{\mathrm{TDM}-\mathrm{IRSR}}=\frac{4 n \sin \theta}{\lambda}
$$

\section{IMPLEMENTATION OF ISOTROPIC RESOLUTION TDM}

To experimentally test this improved configuration, we have designed a special sample rotation system, compatible with high numerical aperture objective and condenser $\left(\mathrm{NA}_{\mathrm{obj}}=\mathrm{NA}_{\mathrm{con}}=1.4\right)$, and we have adapted it onto our existing TDM-IR system [17]. For tomography, the angle of incidence of the illumination beam is controlled using a tip-tilt mirror. The diffracted- and reference beams are recombined in off-axis configuration. Interferograms are corrected for residual aberrations [20], and merged in Fourier space.

We have developed our setup, which is based on a modified Mach-Zehnder interferometer, from Thorlabs, offthe-shelf elements (Figure 2, left). A Thorlabs $10 \mathrm{~mW}$, linear polarization, HNL100L-EC $633 \mathrm{~nm}$ HeNe laser serves as coherent source, and a beam splitter delivers a beam for sample illumination and a reference beam. A B\&W Tek BWB-475-10-OEM 475 nm DPSS laser can also be used. A Newport FSM300 fast steering mirror performs angular scanning illumination, via a high numerical aperture objective (Olympus 100x NA = 1.4 oil immersion), which in fact serves as a condenser. Diffracted field is collected via a high numerical aperture objective (Olympus 100x NA $=1.4$ oil immersion) to form an image onto a PhotonFocus GigE interface, 1280x1024 pixels CMOS camera (PHF-MV1-D2048-96-G2), after recombination with the reference beam.

The acquisition computer triggers the FSM300 tip-tilt mirror, via a LabJack U12 module, and synchronizes the camera acquisitions, to acquire holograms at a 96 frames/s rate. For one 3-D tomographic reconstruction, between 100 and 400 holograms are taken, depending on the desired speed of acquisition and final image quality. An Intel i7, $4 \mathrm{GHz}$ Linux computer, equipped with $32 \mathrm{~Gb}$ of memory performs reconstructions at a typical rate of $3 \mathrm{~s}$ for one 3-D image. A manual z-stage is used for focusing, but, alternately, a purely numerical focusing, by computing hologram propagation, can also be performed.

Our special rotation stage is depicted in Figures 2 middle and right. The difficulty is to perform efficient sample rotation within the narrow space between the condenser-objective, and the detection-objective, which are conventional objectives with a $170 \mu \mathrm{m}$ working distance, and using immersion oil between both objectives to ensure optical contact. A rotating arm is used to insert the sample, which is attached to an optical fibre. A second LabJack U12 module drives a stepper motor, to which the fibre is connected. So, a TDM acquisition with illumination rotation is performed, and then the sample is rotated, and a new TDM dataset with illumination rotation is acquired.

Then, one has to perform an efficient data fusion, in order to take the best of each view, to recompose a final image, with isotropic resolution. At present time, this operation is performed semi automatically, and postacquisition (see Ref. [17], Supplements). 

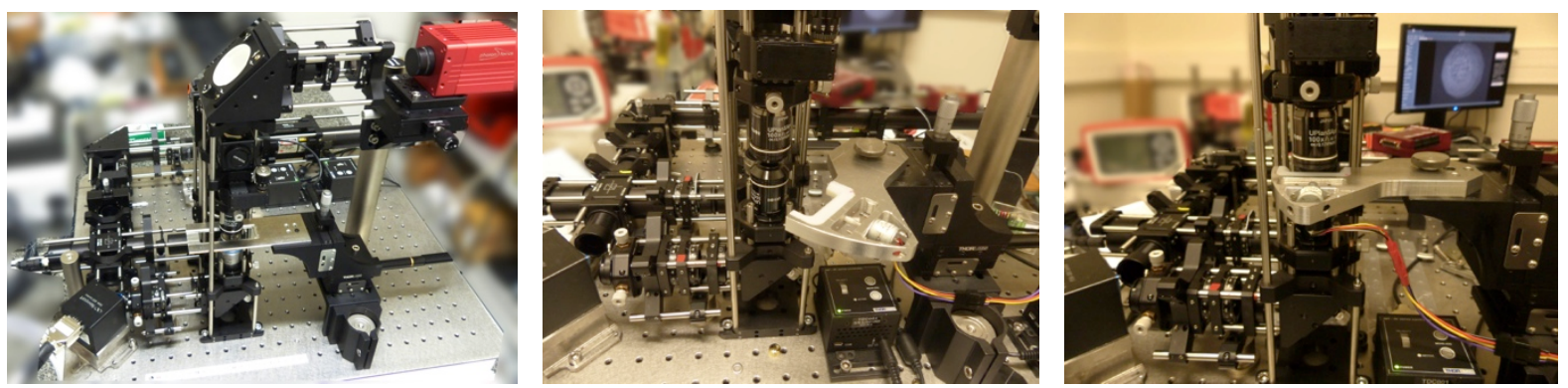

Figure 2. Left: tomographic diffractive microscope built from off-the-shelf elements. Middle: special sample holder to perform specimen rotation. Right: sample engaged between high NA condenser and objective

\section{RESULTS}

We first imaged a tapered optical fibre, obtained with the heat-and-pull technique [18], which produces very sharp tips: typically a few micrometres long, and with a tip end diameter below $100 \mathrm{~nm}$. These characteristics make such an object to be a good test object to estimate the quality gain obtained from different TDM configurations.

Figures 3 Left show lateral, axial and sagittal views of the 3-D image of the fibre tip, obtained with TDM-IR configuration. The $1 \mu \mathrm{m}$-long fibre tip is clearly visible on the lateral view, but the sharp tip is not visible in the lateral view, and even worse, the fibre itself is strongly deformed, and not observed as a cylindrical object on the sagittal view.

Figures 3 Right show the same fibre, but after recombining 4 views, obtained after three successive fibre rotations, for $0^{\circ}, 54^{\circ}, 90^{\circ}$ and $126^{\circ}$. The fibre is now correctly seen as a disc on the sagittal view, and the sharp tip is visible on both axial and on the lateral views. These indicate that the fusion process is efficient, even if not yet perfect, as the lateral resolution is in fact very slightly degraded, but anyway, a large gain in longitudinal resolution is obtained, as expected.
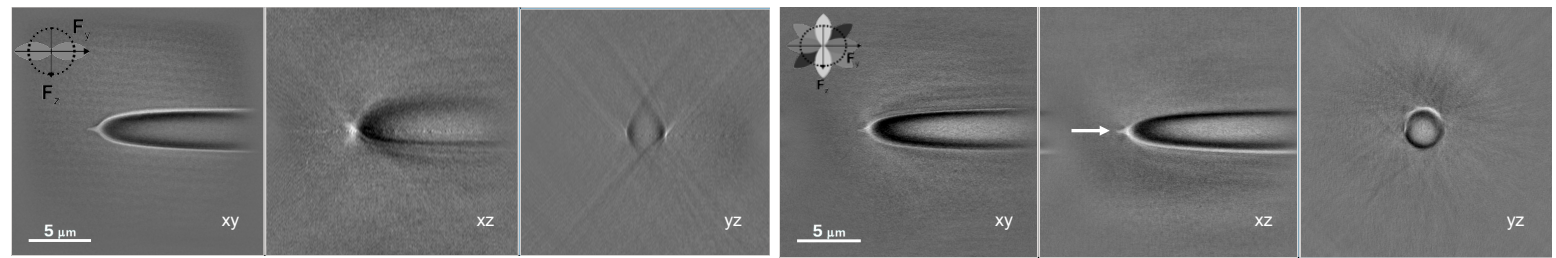

Figure 3. A tapered fibre tip observed with tomographic diffractive microscopy. Left: with TDM-IR. (x-y) lateral

plane view, $(x-z)$ axial plane view, and $(y-z)$ sagittal view. Right: same fibre observed with TDM-IR-SR.

We have then developed a specific procedure to attach samples, like pollens, diatoms, or microcrystals, to an optical fibre [17]. The fibre is therefore in that cased used as both sample holder and sample rotator for TDMIRSR. Figures 4 displays a zeolite crystal imaged with one view (TDM-IR) on Fig. 4(a), and after fusion of 8 acquisitions (TDM-IRSR) on Figs. 4(b-e). The crystal is much better imaged, and note that the microbubbles surrounding the crystal appear noticeably elongated on Fig. 4(a), while they are spherical on Figs. 4(b-e).

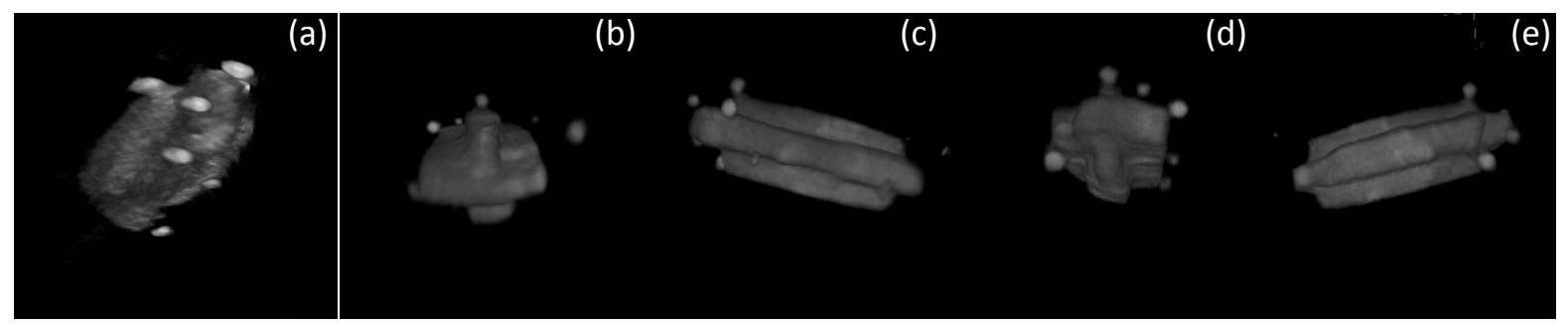

Figure 4. (a): Image of a zeolithe microcrystal observed with TDM-IR (1 angle of TDM-IRSR). (b-e) same crystal observed with TDM-IRSR, combining 8 views of the sample.

\section{CONCLUSION}

We have developed an advanced tomographic diffractive microscope, which combines illumination and specimen rotations. Such a system delivers images with an isotropic and improved resolution, unreachable by other (non-fluorescence) optical microscopy approaches. The obtained images also allow for clear distinction of refraction and attenuation, which could help distinguishing cellular substructures [17][19]. More details about the method can be found in Ref. [17] and its supplements. 
We believe that biological research on freestanding samples (microplankton science, palynology, bacteriology, hematology, gamete and fertilization studies), as well as research on transparent materials like photopolymers, structured optical fibres, textile fibre science, micro- and nanofabrication characterization, microcrystal could benefit from the superior imaging capabilities of such an instrument.

A limitation of our system at present time is tedious sample manipulation. However, promising-, contact-free sample rotation systems are now under development [20]-[23], and could facilitate the use of this technique with living, freestanding samples, like red/white blood cells, egg cells, pollens, or unicellular organisms.

\section{REFERENCES}

[1] Myung K. Kim, Digital Holographic Microscopy: Principles, Techniques, and Applications, Springer Series in Optical Science Vol. 162, Springer, New York, 2011.

[2] G. Popescu, Quantitative Phase Imaging of Cells and Tissues, McGraw Hill, New York, 2011.

[3] K. Lee, K. Kim, J. Jung, J. Heo, S. Cho, S. Lee, G. Chang, Y. Jo, H. Park, and Y. Park, Quantitative Phase Imaging Techniques for the Study of Cell Pathophysiology: From Principles to Applications, Sensors, vol. 13 pp. 4170-4191, 2013.

[4] B. Kemper, Digital holographic microscopy: quantitative phase imaging and applications in live cell analysis, in Handbook of Coherent-Domain Optical Methods, V. V. Tuchin, ed., pp. 215-257, Springer, New York, 2013.

[5] B. Bhaduri et al., Diffraction phase microscopy: principles and applications in materials and life sciences, Adv. Opt. and Phot., vol. 6 pp. 57-119, 2014.

[6] O. Haeberlé et al., Tomographic Diffractive Microscopy: Basics, Techniques and Perspectives, J. Mod. Opt., vol. 57 pp. 686-699, 2010.

[7] E. Wolf, Three-dimensionnal structure determination of semi-transparent objects from holographic data, Opt. Comm., vol. 1 pp. 153-156, 1969.

[8] V. Lauer, New approach to optical diffraction tomography yielding a vector equation of diffraction tomography and a novel tomographic microscope, J. Microscopy, vol. 205 pp. 165-176, 2002.

[9] W. Choi et al., Tomographic phase microscopy, Nature Meth., vol. 4 pp. 717-719, 2007.

[10] M. Debailleul et al., Holographic microscopy and diffractive microtomography of transparent samples, Meas. Sci. Technol. Vol. 19074009 (8pp.), 2008.

[11] M. Debailleul et al., High resolution three-dimensional tomographic diffractive microscopy of transparent inorganic and biological samples, Opt. Lett., Vol.34 pp. 79-81, 2009.

[12] Y. Cotte, et al., Marker-free phase nanoscopy, Nature Phot., vol. 7 pp. 113-117, 2013.

[13] S. Vertu et al., Improved and isotropic resolution in tomographic diffractive microscopy combining sample and illumination rotation, Centr. Eur. J. of Phys.,vol. 9, pp. 969-974, 2011.

[14] S. Vertu et al., Diffraction microtomography with sample rotation: influence of a missing apple core in the recorded frequency space, Centr. Eur. J. of Phys., vol. 7 pp. 22-31, 2009.

[15] Y.-C. Lin, and C.-J. Cheng, Sectional imaging of spatially refractive index distribution using coaxial rotation digital holographic microtomography, J. Opt., vol. 16065401 (8 pp.), 2014.

[16] M. Kujawińska et al., Problems and Solutions in 3-D Analysis of Phase Biological Objects by Optical Diffraction Tomography, Int. J. of Optomechatronics, vol. 8 pp. 357-372, 2014.

[17] B. Simon, et al:: Tomographic diffractive microscopy with isotropic resolution, Optica vol. 4 pp. 460-463, 2017

[18] M. Xiao et al., Simple device for making optical fiber tips for scanning near field optical microscopes, Rev. Sci. Instrum., vol. 68 pp. 2787-2789, 1997.

[19] B. Simon, et al., High resolution tomographic diffractive microscopy of biological samples, J. Biophotonics, vol. 3 pp. 462-467, 2010.

[20] M. K. Kreysing et al., The optical cell rotator, Opt. Exp., vol. 16 pp. 16984-16992, 2008.

[21] M. Habaza et al., Tomographic phase microscopy with $180^{\circ}$ rotation of live cells in suspension by holographic optical tweezers, Opt. Lett., vol. 40 pp. 1881-1884, 2015.

[22] B. Le Saux et al., Isotropic High-Resolution Three-Dimensional Confocal Micro-Rotation Imaging for Non-Adherent Living Cells, J. Microsc., vol. 233 pp. 404-416, 2009.

[23] M. Habaza et al., Rapid 3D refractive-index imaging of live cells in suspension without labeling using dielectrophoretic cell rotation, Adv. Sci., vol 41600205 (9 pp.), 2016. 